\title{
Symptoms of testosterone deficiency in early middle aged men
}

Anna-Clara Spetz, Mats Fredrikson and Mats Hammar

\section{Linköping University Post Print}

N.B.: When citing this work, cite the original article.

Original Publication:

Anna-Clara Spetz, Mats Fredrikson and Mats Hammar, Symptoms of testosterone deficiency in early middle aged men, 2012, The Aging Male, (15), 2, 78-84.

http://dx.doi.org/10.3109/13685538.2012.669435

Copyright: Informa Healthcare http://informahealthcare.com/

Postprint available at: Linköping University Electronic Press http://urn.kb.se/resolve?urn=urn:nbn:se:liu:diva-78263 


\section{Symptoms of Testosterone deficiency in early middle aged men.}

Anna-Clara E Spetz Holm, MD, PhD, Mats G Fredrikson*, PhD, and Mats L Hammar, MD, $\mathrm{PhD}$.

Division of Obstetrics and Gynecology, Department of Clinical and Experimental Medicine, Faculty of Health Sciences, Linköping University, Department of Obstetrics and Gynecology in Linköping, County Council of Östergötland, Linköping, Sweden *Division of Occupational and Environmental Medicine, Department of Clinical and Experimental Medicine and Linköping Academic Research Center, Faculty of Health Sciences, Linköping University.

Short title: Symptoms of low T and BT

Key words: men; andrology; climacteric; questionnaires; testosterone; androgens

\section{Reprint requests and correspondence:}

Anna-Clara Spetz Holm, Department of Clinical and Experimental Medicine, Obstetrics and Gynecology, Faculty of Health Sciences, University Hospital, S-581 85 Linköping, Sweden.

e-mail: Anna-Clara.Spetz.Holm@LiU.se Phone: +46 101033130, Fax: +4613148156 


\begin{abstract}
:
Symptoms of testosterone deficiency and concentrations of testosterone (T) and Bioavailable Testosterone (BT) were studied in 35 and 45 year old men.

Methods: A questionnaire, was sent to all 35 and 45 year old men in Linköping, Sweden $(\mathrm{n}=1998)$. The questionnaire has earlier been used for 55-75 year old men and included demographic data, medical history, different symptoms possibly of testosterone deficiency and the 10 questions from the "ADAM-questionnaire". Totally 200 men randomly selected among the men who answered the questionnaire were asked to give blood samples for analysis of Tand BT-concentrations.
\end{abstract}

Results: A total of $38.7 \%$ of the questionnaires were returned and analysed, and $43.5 \%$ of the 200 randomly selected men gave blood samples. The older age group reported more symptoms that may be connected to low B and BT and had lower T- and BT-concentrations. Less strong erections and higher alcohol consumption were associated with lower concentrations of BT in 45 year old men.

Conclusions: The burden of symptoms possibly related to low T concentrations were higher in 45 years old men, and BT and T were lower. However, due to the low answer frequency and number of blood samples analyzed no general conclusions can be drawn. 


\section{Introduction:}

The production of testicular testosterone gradually decreases with age in men but with individual variation [1]. In normally aging men, total testosterone and albumin-bound testosterone concentrations in serum decline, whereas sex hormone binding globulin (SHBG) usually increases [2]. This leads to a more readily observable decrease in circulating free or bio-available testosterone (BT) than in total testosterone (T) [1]. However, the decline in androgens seems to begin earlier than had previously been believed, i.e. at approximately age 30 , and the decline is progressive [3].

Partial Androgen Deficiency in Aging Males (PADAM) or Androgen Deficiency in Aging Males (ADAM) [4] and Late Onset Hypogonadism (LOH) [5] or Testosterone Deficiency Syndrome (TDS) [6] are expressions all used to describe the gradual decline in androgens in men. This decline is sometimes associated with a number of rather subtle symptoms including gradual loss of muscle strength and bone density, decrease in libido, memory, enjoyment of life and mood, as well as impotence, asthenia and cognitive dysfunctions [7-10]. PADAM continues to be a controversial concept since the symptoms considered in PADAM may also be caused by the normal aging per se or due to concomitant diseases.

We have previously reported that hot flushes, similar to those experienced by women during the climacteric period, are quite common in a population of ageing Swedish men, consisting of totally almost 1400 men, 55, 65 and 75 years old, and that the flushes cause significant discomfort for those men who report them [11].

In a cross-sectional study of men in Sweden, performed in 2003, we investigated the prevalence of probable symptoms of $\mathrm{LOH}$ and associated them with testosterone concentrations [12]. We found that symptoms associated with LOH often occur in an elderly population, but we could only find an association between three symptoms and blood testosterone concentrations, one being bothersome hot flushes why we suggest that hot flushes 
should be addressed as a possible LOH-symptom. Also in line with previous reports, $4.5 \%$ of men reported hot flushes. To follow changes in LOH-symptoms as well as testosterone concentrations, we used the same cross-sectional study design as in 2003 in the same cohort of men five years later [13]. We found that both $\mathrm{T}$ and BT decreased over the five year period, and also that the patients' "symptom burden" had increased. We could not, however, associate the decline in androgens with the increase of specific symptoms over the five year period. We also found that LOH symptoms were more common in older men [13].

In line with our study, other studies $[14,15]$ have failed to identify a connection between LOH symptoms and circulating androgen levels. It is likely that symptoms associated with LOH have a complex background and are not caused simply by partial androgen deficiency. It might be that changes in androgen concentrations over time are more important for development of LOH-symptoms than the actual androgen levels [13].

Since we have previously investigated possible symptoms of LOH and measured T and BT in men 55 to 75 years old [12,13], and since the decline in androgens seems to begin earlier than previously believed, i.e. at approximately age 30 [3], we wanted to investigate symptoms of testosterone deficiency and androgen concentrations in a an even younger group of men. We therefore used the same questionnaire as in the former studies of men 55 to 75 years old, but now turned to men aged 35 and 45 years, and also asked 200 randomly selected men to give blood-samples for analyses of T and BT concentrations. 


\section{Methods:}

\section{Participants:}

A questionnaire was sent in 2010 to all men age 35 or 45 years $(n=2033)$, living in the community of Linkoping, Sweden during 2010. The questionnaire included questions about demographic data, marital status, medical history, mood disorders, ongoing medication including hormone therapy, alcohol, smoking, exercise habits etc. The question about hot flushes asked if the subject experienced a sudden and unexpected feeling of warmth or sweating unrelated to being in a warm environment or doing physical work. The men were asked about current or former hot flushes, as well as the extent to which they were bothered by them. They were also asked about fatigue, muscular/skeletal pain, balance, frailty fractures, decreased libido, decreased energy and changes in muscular strength/endurance among other questions. A random sample of the men, who, in the questionnaire, had agreed to give a blood sample for analyses of Testosterone, Albumin, SHBG and Albumin were contacted and were again asked to give a blood sample. Body Mass Index (BMI) was calculated for each individual by asking for height and weight.

The questionnaire was designed to make it as easy as possible for the subjects to answer. All that had to be done was to make a tick mark in one of a series of boxes. The questionnaire was slightly modified from a previously used and validated questionnaire developed by us $[11,16]$. Reproducibility was tested by Spetz et al 2007 [12 ].

\section{The ADAM-questionnaire}

Morley et al. have validated the use of the ADAM-questionnaire, which includes questions on 10 symptoms, as a means of screening for individuals with possible low levels of BT $[17,18]$. The 10 questions from the ADAM-questionnaire developed at St. Louis University Medical 
School, Canada, were translated into Swedish and scattered throughout the questionnaire. According to Morley and co-workers, answering "yes" to item 1 or 7 or "yes" to any other three questions predicts low BT with a sensitivity of $88 \%$ and a specificity of $60 \%$ [18]. Therefore we made a score; "ADAM-positivity and -negativity", respectively, meaning that men positive for the ADAM-questionnaire (answering "yes" to item 1 or 7 or "yes" to any other three questions or more) were "ADAM-positive", and all other men were "ADAMnegative" [12]. We also developed a term called "ADAM-sum", according to the number of "yes" replies in the questionnaire. One to three "yes" is called "low ADAM-sum", four to six "yes" is called "medium ADAM-sum" and seven to ten "yes" is called "high ADAM-sum", respectively. In this case we did not give the answers to questions 1 or 7 any special attention [12].

\section{Data handling and statistics}

The questionnaires were coded only in order to be able to send a reminder to the subjects. All information from the answered questionnaires was optically scanned and exported to SPSS for Windows. The agreement between optical and manual reading was checked for the first ten complete questionnaires and the optical reading was continued only after the manual and the optical reading agreed totally. All information was handled confidentially. All data were analysed by STATA 11.1 (College Station, Tx, USA). To test the differences between agegroups we used Pearson $\chi^{2}$ and Fisher's exact test. A p-value $<0.05$ was considered as significant. ANOVA-, with Bonferroni correction for post hoc tests-, and Students t-test were used when appropriate to find differences between grades of symptoms/single symptom and BT and T, respectively. Regression analyses were used to investigate if any associations were found between different symptoms and BT and T. Hierarchical cluster-analysis was used to investigate whether a number of symptoms correlated with each other and /or BT, or not. 
Laboratory investigations:

Albumin was analysed using Immunochemistry (Advia 1800, Siemens Laboratory

Diagnostics, Sweden). SHBG was measured using electrochemiluminiscence immunoassay (ECLIA) on cobas ${ }^{\circledR}$ e 602, (Roche Diagnostics, Sweden). T was analysed using ECLIA on cobas ${ }^{\circledR}$ e 602, Roche Testosterone II ( $2^{\text {nd }}$ generation) Roche Diagnostics, Sweden. All assays were performed at the department of Clinical Chemistry, University Hospital, Linkoping. BT was calculated based on concentrations of T, Albumin, and SHBG according to an algorithm previously described [19]. 


\section{Results}

A total of 2033 questionnaires were sent out and 35 were returned by the postal service due to unknown address; thus the study population consisted of 1998 men. After one reminder, 788 questionnaires were returned, of which 14 were not filled in at all, and one patient had returned and filled in the questionnaire twice and returned both. Thus 773 questionnaires $(38.7 \%)$ were eligible for analysis, about the same percentage in the two age groups. We received letters from an additional 11 men giving different reasons for not wanting to participate in the study.

The men 35 years old ( 35 YY) constituted $44.8 \%$ of the study population $(n=346)$ and the 45 YY men 55.2\% (n=427). Demographic and other data are presented in Table I. The two groups were similar except that the younger group was leaner, smoked to a lesser extent and jogged to a higher extent (Table I).

Only one man in the study, a 35 year old man had undergone androgen ablation for an unknown reason. Diabetes was reported by two of the 35 YY men and 9 of the 45 YY men. Cardiovascular disease was reported by six men in the 45 YY group. Hypertension was reported by five men in the 35 YY group and by 23 of the $45 \mathrm{YY}$ group and three and two men in $35 \mathrm{YY}$ and $45 \mathrm{YY}$, respectively, had prostate problems. In total four men reported cancer; two testicular cancers, one lymphoma and one colon cancer. Psychiatric disorders were reported by eight 35 YY and 1245 YY men. Daily medication was used by $12.0 \% 35$ YY men and $21.0 \%$ of 45 YY men $(\mathrm{p}=0.001)$. Thirty-two men (5.0\%) used medication for hypertension and/or cardiac disease. None used Warfarin, six men used blood-lipid-lowering medication. Psychoactive drugs, e.g. selective serotonin reuptake inhibitors (SSRI) and serotonin and norepinephrine reuptake inhibitors (SNRI) were used by 21 men and one used benzodiazepines. Sleeping pills were used by six men. Daily intake of psychoactive medicine was reported by 43, but only 28 men specified which drug/drugs they were taking. Five men used chronic medication for pain-relief more than paracethamol. Other medications $(n \leq 4)$ included insulin, oral anti diabetic medication, medication for asthma or allergy, constipation and epilepsy. 
Table I: Demographic and clinical data on men answering the specific questions (mean +/- SD or percentage(n)). Answers to every item were not given in all questionnaires.

Age group:

$\begin{array}{ll}35 \mathrm{YY} & 45 \mathrm{YY} \\ \mathrm{n}=346 & \mathrm{n}=427\end{array}$

BMI $\left(\mathrm{kg} / \mathrm{m}^{2}\right)$

$25.2 \pm 0.16$

$26.2 \pm 0.19$

$\mathrm{p}<0.001$

$\mathrm{n}=344$

$\mathrm{n}=422$

Increased waist-size

$45.3 \%(139)$

$62 \%(225)$

$\mathrm{p}<0.0001$

Smoking status

Never smoked

$81.1 \%(279)$

$69.3 \%(293)$

$\mathrm{p}<0.05$

Former smoker

$14.8 \%(51)$

$19.39 \%(82)$

$\mathrm{p}<0.05$

Smoker

$4.1 \%$ (14)

$11.4 \%(48)$

$\mathrm{p}<0.05$

Alcohol use

No alcohol

$8.7 \%(30)$

$12.0 \%(51)$

ns

Some times/month

$46.8 \%$ (162)

$41.3 \%$ (175)

ns

Every week

$44.5 \%$ (154)

$46.0 \%$ (195)

ns

Every day

$0.39 \%$ (3)

ns

Employ status:

Work fulltime

$90.1 \%(308)$

$87.1 \%$ (370)

ns

Work part-time

$4.4 \%(15)$

$4.0 \%$ (17)

ns

Retired

$$
0.3 \% \text { (1) }
$$

Applying for job

$4.4 \%(15)$

$5.9 \%(25)$

ns

Sick-leave

$0.6 \%$ (2)

$1.2 \%(5)$

ns

Retired due to disease

$0.3 \%(1)$

$1.9 \%(8)$

ns

Civil status:

Married/co-habiter

$78.9 \%(273)$

$77.5 \%(330)$

ns

Living alone,

Previously married/co-habiter

$6.65 \%(23)$

$10.1 \%(43)$

ns

In a relationship

but not living together

$4.1 \%(14)$

$3.8 \%(16)$

ns

Living alone

$10.4 \%(36)$

$8.7 \%(37)$

ns

Exercisehabits: 
Golf

Regular walks

Bicycling

Tennis, table tennis,

Badminton or squash

Jogging

Workout/swimming

Other sport activities

Degree of physical activity at work

Immobile work activities

Varied and mobile

Physical heavy
$57.7 \%(198)$

$25.8 \%(42)$

$79.6 \%(203)$

$55.9 \%(113)$

$31.1 \%(52)$

$58.9 \%(132)$

$24.7 \%(39)$

$72.0 \%(159)$

$33.8 \%$ (116)

$8.5 \%(29)$
$28.2 \%(61)$

ns

$80.7 \%(271) \quad$ ns

$57.6 \%(148)$

ns

$\mathrm{p}<0.005$

$\mathrm{p}<0.05$

ns

ns

$40.3 \%(171) \quad \mathrm{ns}$

$6.6 \%(28) \quad \mathrm{ns}$

None used Testosterone-gel or -injections. Medications for unspecified erectile dysfunction were currently used or previously tried by $2.7 \%$ of all men.

\section{Laboratory tests}

Of the study population, 537 men accepted the invitation to give a blood-sample for testosterone analyses. Out of these 537 men 100 men from each of the 35 YY and 45 YY groups of men were randomly selected and invited by letter to give non-fasting morning (before $11 \mathrm{AM}$ ) serum samples at their nearest Primary Health Care Centre. After six weeks one reminder was sent to the men that had not given blood-samples ( $\mathrm{n}=145)$. Blood-samples were given by a total of 90 men (45\%) after one reminder; however the blood-samples of three men were lost before analysis, i.e. blood samples were analysed from 87 men $(43.5 \%)$. Hormonal data are presented in Table II, showing that BT- and Tconcentrations were significantly lower in the older age group $(\mathrm{p}<0.001$ and $\mathrm{p}<0.005)$. $\mathrm{T}$ concentrations were classified into three different groups; i.e. $<8 \mathrm{nmol} / \mathrm{L}(\mathrm{n}=5), 8-11.99 \mathrm{nmol} / \mathrm{L}$ $(\mathrm{n}=19)$ and $\geq 12 \mathrm{nmol} / \mathrm{L}$ ( $\mathrm{n}=63$ ), according to guidelines of testosterone deficiency [20]. BT concentrations were grouped into three categories (equal thirds) according to the number of the men; the lower third was called "low BT" (mean $5.35 \pm 0.70 \mathrm{nmol} / \mathrm{L}, \mathrm{n}=30$ ), the middle third "median BT" 
(mean $7.36 \pm 0.53 \mathrm{nmol} / \mathrm{L}, \mathrm{n}=28)$ and the higher third of the men was called "high BT" (mean $9.64 \pm$ $1.20, n=29)$.

Table II: Measurements of hormones in different age-groups

\begin{tabular}{|c|c|c|c|c|c|}
\hline & Age group: & $35 \mathrm{YY}$ & $45 \mathrm{YY}$ & All men & \\
\hline & & $\mathrm{n}=45$ & $\mathrm{n}=42$ & $\mathrm{n}=87$ & $\mathrm{p}$ \\
\hline BT (nmol/L) & & $8.10 \pm 1.91$ & $6.70 \pm 1.78$ & $7.42 \pm 1.97$ & $<0.001$ \\
\hline $\mathrm{T}(\mathrm{nmol} / \mathrm{L})$ & & $16.9 \pm 6.46$ & $13.2 \pm 4.63$ & $15.1 \pm 5.92$ & $<0.005$ \\
\hline SHBG (nmol/L) & & $37.8 \pm 17.7$ & $31.8 \pm 12.5$ & $34.9 \pm 15.6$ & $\mathrm{~ns}$ \\
\hline Albumin (g/L) & & $44.0 \pm 2.47$ & $43.2 \pm 2.52$ & $43.6 \pm 2.51$ & ns \\
\hline
\end{tabular}

$\mathrm{BT}=$ Bioavailable Testosterone; $\mathrm{T}=$ Testosterone; $\mathrm{SHBG}=$ Sexual Hormone Binding Globulin

Men with BMI less than $25 \mathrm{~kg} / \mathrm{m}^{2}$ had higher BT $(8.15 \pm 2.09 \mathrm{nmol} / ; \mathrm{n}=32)$ than overweight men, with $\mathrm{BMI} \geq 25-29.9 \mathrm{~kg} / \mathrm{m}^{2}(\mathrm{BT} ; 7.02 \pm 1.88 \mathrm{nmol} / \mathrm{L} ; \mathrm{n}=48, \mathrm{p}<0.05)$. No significant differences were seen in BT in overweight compared to obese men $\left(\mathrm{BMI} \geq 30 \mathrm{~kg} / \mathrm{m}^{2}\right)$, probably because of the small number of obese men $(n=7)$.

\section{Alcohol and BT}

In 45 YY men reporting alcohol intake at least a couple of times per week, BT was significantly lower $(6.14 \pm 0.34 \mathrm{nmol} / \mathrm{L}, \mathrm{n}=24)$ compared to men who used alcohol less often (one to a couple of times every month; $7.44 \pm 0.40 \mathrm{nmol} / \mathrm{L}, \mathrm{n}=18 ; \mathrm{p}=0.017) .35 \mathrm{YY}$ who drank alcohol at least once a week had higher BT, according to the categories BT ("high BT" and "median BT") compared to men who used alcohol more seldom that had "low BT" ( $\mathrm{p}=0.0013$ and $\mathrm{p}=0.0017)$. In $45 \mathrm{YY}$, the opposite was found, $66.6 \%$ of men who drank alcohol a couple of times every week or more had "low BT". This was 
significant compared to men with "median BT" ( $\mathrm{p}=0.017$, Table III). No differences were seen for $\mathrm{T}$ and alcohol use.

Table III: Alcohol intake in relation to BT-categorises*.

$35 \mathrm{YY}$

\begin{tabular}{llll}
\hline & "low BT" & "median BT" & "high BT" \\
Alcohol intake** & & & \\
< weekly & $33.3 \% ; \mathrm{n}=9$ & $22.2 \% ; \mathrm{n}=6$ & $44.4 \% ; \mathrm{n}=12$ \\
> weekly & $\mathrm{n}=0$ & $38.9 \% ; \mathrm{n}=7$ & $61.1 \% ; \mathrm{n}=11$
\end{tabular}

$45 \mathrm{YY}$

Alcohol intake**

"low BT"

"median BT"

"high BT"

$<$ weekly

$27.8 \% ; n=5$

$55.6 \% ; \mathrm{n}=10$

$16.7 \% ; \mathrm{n}=3$

$>$ weekly

$66.7 \% ; n=16$

$20.8 \% ; n=5$

$12.5 \% ; n=3$

*BT concentrations were divided in three categories (thirds) based on the number of the men.

BT category; "low BT" (mean $5.35 \pm 0.70 \mathrm{nmol} / \mathrm{L}$ )

BT category; "median BT" (mean $7.36 \pm 0.53 \mathrm{nmol} / \mathrm{L}$ ) and

BT category; "high BT" (mean $9.64 \pm 1.20$ ).

** "< weekly"; alcohol one to a couple of times every month

"> weekly"; alcohol intake a couple of times per week or more

\section{Less strong erection and $B T$}

All 45 YY men who reported the symptom " less strong erections" had "low BT" $(25 \% ; n=2)$ or "median BT" $(75 \% ; n=6)$. However, $82.4 \%(n=28)$ of the men who did not report problems with their erections also had "low BT" or "median BT". In 35 YY men there were no differences concerning Tor BT-concentrations in relation to problems with erections.

\section{Exercise habits and the muscular/skeletal system}

On the average, $35 \mathrm{YY}$ men spent $14.9 \pm 0.84$ hours/month on physical exercise and $45 \mathrm{YY}$ men spent $14.4 \pm 0.55$ hours on physical activity. More than half of the men $(55.4 \%)$ exercised at least 12 hours/month. In total 69 men (9\%) never engaged in any regular physical activity. However, there were no differences between age groups or in T- or BT-concentrations in men 
with different degrees of activity. The type and frequency of exercise habits are presented in Table I.

Of all men, $4.6 \%$ reported difficulties with their balance some times/month or more, $0.9 \%$ had daily complaints. $2.2 \%$ of all men reported that they had fallen at least once in an uncontrolled manner during the past year. T- and BT-concentrations did not differ between men who reported balance problems and falls and those who did not.

Fractures at osteoporotic sites, i.e. fracture of the wrist, neck of the femur or vertebra were reported by $7.0 \%$ of all men but there was no difference between age-groups or in relation to T- and BTconcentrations.

\section{Hot flushes}

Hot flushes at least a few times weekly or more were reported by $4.3 \%(n=33)$ and were more often reported among $45 \mathrm{YY}(5.9 \%, \mathrm{n}=25)$ than $35 \mathrm{YY}$ men $(2.3 \% ; \mathrm{n}=8, \mathrm{p}<0.05)$. The duration of the symptom was 3.8 years $(n=6)$ in 35 YY men and 4.2 years $(n=20)$ in 45 YY men.

Of men reporting hot flushes at least a few times weekly $78.8 \%$ were bothered by them, and $45.5 \%$ suffered severely. T and BT concentrations did not differ either between age groups or between men who reported and/or suffered from hot flushes and those who did not.

There was no significant difference in the prevalence of hot flushes between those men who exercised more than 12 hours per month and those who exercised less or between men who used alcohol once a week or more and men who used alcohol once a month or less. About $18.6 \%(n=8)$ of men using psychoactive drugs reported hot flushes at least a few times weekly compared to $3 \%(n=18)$ of men not using psychoactive drugs $(\mathrm{p}<0.0001)$. Using psychoactive drugs increased the risk of having flushes about 7 times $(\mathrm{OR}=7.36)$.

\section{The ADAM-questionnaire and other possible symptoms of LOH}

Six of ten symptoms from the ADAM-questionnaire were more common in $45 \mathrm{YY}$ men than in $35 \mathrm{YY}$ men (Table IV). Dry eyes and muscular/skeletal pain were more often reported by 45 YY men than by 35 YY men (p<0.05). More 45 YY men were ADAM-positive (53.7\%; $\mathrm{n}=190)$ than 35 YY $(42.4 \%$; 
Table IV: Different symptoms in relation to the age groups. (Chi-square-test).

$$
\begin{array}{lll}
\text { Age group: } & 35 \mathrm{YY} & \begin{array}{l}
45 \mathrm{YY} \\
\mathrm{n}=342-346 \quad \mathrm{n}=419-426
\end{array}
\end{array}
$$

Decrease in libido*

Lack of energy*

Decreased strength and/or endurance*

Loss in height*

Decreased enjoyment of life*

Sadness and/or grumpiness*

Less strong erections*

Deterioration in ability to play sports*,**

Falling asleep after dinner*

Deterioration in work performance*

Dry eyes

Dry and/or fragile skin

Muscular/skeletal pain

Decreased need to shave

Hot flushes:

a few times a week or more
$18.3 \%(63)$

$36.2 \%(125)$

$41.0 \%(141)$

$3.5 \%(12)$

$23.4 \%(81)$

$24.6 \%(85)$

$12.5 \%(43)$

$18.9 \%(60)$

$10.8 \%(37)$

$23.9 \%(82)$

$9.9 \%(34)$

$31.6 \%(303)$

$17.8 \%(61)$

$2.9 \%(10)$
$23.0 \%(98)$

$44.3 \%$ (188)

$47.4 \%$ (201)

$10.7 \%(45)$

$26.5 \%(113)$

$26.5 \%(112)$

$19.5 \%(83)$

$27.8 \%(105)$

$18.5 \%(79)$

$37.9 \%$ (159)

$14.9 \%(63)$

$28.2 \%$ (119)

$24.9 \%$ (106)

$5.4 \%(23)$

$5.9 \%(25)$ ns

$\mathrm{p} \leq 0.05$

ns

$\mathrm{p}<0.0001$

ns

ns

$\mathrm{p}<0.01$

$\mathrm{p}<0.01$

$\mathrm{p}<0.005$

$\mathrm{p}<0.0001$

$\mathrm{p}<0.05$

ns

$\mathrm{p}<0.05$

ns

*A question included in the ADAM-questionnaire

**Only of all men that ever have exercised regularly, i.e. 31735 YY and $37845 Y Y$

$\mathrm{n}=129 ; \mathrm{p}=0.004)$. According to ADAM-sum, $45 \mathrm{YY}$ men had higher scores, i.e. higher symptom burden, (ADAM-sum mean $2.68 \pm 0.13$ ) than $35 \mathrm{YY}$ (ADAM-sum mean $2.10 \pm 0.13 ; \mathrm{p}=0.0014$ ). No matter if the men were classified into ADAM-positive/negative or "low", medium" or "high" ADAM sum did we find differences in $\mathrm{T}$ or BT between the groups.

Extensive cluster analyses were made to try to group symptoms, but no specific symptom complex of importance was found.

Comparisons of symptoms and hormones with men from an own previous study [12] 
BT did not differ between 35 or $45 \mathrm{YY}$ men in the current study compared to $55-59,60-65$ or 66-75 YY men [12]. However, T-concentrations were lower in $45 \mathrm{YY}$ old than in 55-59, 60-65 and 66-75 YY men from the previous study $(\mathrm{p}<0.05)$.

The percentage of men with "high ADAM-sum" increased with age, from $3.0 \%$ in $35 \mathrm{YY}$ men to $25.0 \%$ in $65 \mathrm{YY}$ men but decreased in $75 \mathrm{YY}$ men to $19 \%$ ( $\mathrm{p}<0.05$ when comparing $65 \mathrm{YY}$ and $75 \mathrm{YY}$ men).

\section{Discussion}

The $\mathrm{T}$ and BT concentrations were significantly lower in 45YY men than in $35 \mathrm{YY}$ men and 6 of 10 symptoms from the ADAM-questionnaire were more common in the older age group. The men in group $35 \mathrm{YY}$ were taking part in sports more often, were leaner, smoked less and had a lower daily intake of medicine than 45 YY men. Despite these differences, no statistical associations were found between single symptoms and T- and BT-concentrations even after dividing men into categories based on T-concentrations where we used cut off values according to the current guidelines for the diagnosis of testosterone deficiency [20]. Hot flushes at any intensity were reported by $33.1 \%$ of non-castrated men 55, 65 and 75 years old men in an earlier study, using the same questions as in the current study, $4.3 \%$ at least a few times/week. More frequent flushes were reported by 75YY than 55 YY men [11]. In the current study, 26.9\% reported hot flushes and 3.7\% at least a few times/week, and in line with the former study [11], half of the men were also bothered by the flushes. It seems that the occurrence of hot flushes is less common in the younger age groups. However, of the men that reported hot flushes in the current study, half of them were also bothered by them. This is in line with earlier studies of men [11-13]. It was difficult to perform detailed comparisons between other LOH-symptoms and hot flushes since there were only a few men in the current study reporting flushes at least a few times/week. However, it appears that the risk of having flushes increases with concurrent treatment with SSRI, SNRI and/or sleeping pills. As is well 
known, these medications can be used as treatment for hot flushes [21], but can also induce them.

The diagnosis of $\mathrm{LOH}$ is complex, and efforts have been made to develop a reliable screening instrument [18, 22-24], but the limited specificity of the available instruments make them difficult to use for general screening [20]. The difference in age of those showing the first symptoms of testosterone deficiency and those showing LOH is also unclear, since LOH develops during "advanced age". There is no clear definition of "advanced age", but from the context of the recommendations it may be interpreted as referring to men over $60 \mathrm{YY}$ [20]. Therefore, we find the expression "symptoms of testosterone deficiency" or "hypogonadism" is to be regarded as the more correct term for this study group of $35 \mathrm{YY}$ and $45 \mathrm{YY}$ men. Many research groups, including ours $[12,13]$ have tried to find "single" or "a combination" of symptoms that can specifically be used as diagnostic criteria of $\mathrm{LOH}$, without being obscured by nonspecific symptoms of aging. In a large multicentre European study, 3219 men 40-79 YY were investigated for LOH symptoms and measurements of T and BT. However, the mean age was 59.7 years, and the adjusted mean response rate at each centre was $43 \%$. They stated that the presence of the three sexual symptoms was clustered with a low total testosterone level, (i.e. less than $11 \mathrm{nmol} / \mathrm{L}$ ); "decreased frequency of morning erections", "decreased frequency of sexual thoughts" and "erectile dysfunction" [25]. In a study by Araujo et al $4.2 \%$ of men younger than $50 \mathrm{YY}$ were diagnosed with symptomatic androgen deficiency and $8.4 \%$ of men 50+ YY [26]. Men were considered symptomatic if they had one of the specific sexual symptoms (low libido, erectile dysfunction, or osteoporosis) or two or more of the nonspecific symptoms (sleep disturbance, depressed mood, lethargy, or low physical performance), in line with guidelines that are proposed by The Endocrine Society [27]. In our questionnaire, only one of these three symptoms was asked for; erectile dysfunction; however in a milder form expressed by the phrase "less strong erections" as used in the ADAMquestionnaire. A significantly greater percentage of $45 \mathrm{YY}$ men than $35 \mathrm{YY}$ men reported this 
symptom and in 45 YY men all who reported this symptom had "low BT" or "median BT". It was shown, however, that more than $80 \%$ of men without this symptom also had BT in the same level, indicating that this symptom has many causes other than low BT. In Sweden, there is no tradition of testosterone therapy, which was also confirmed in the current study where none of the participants had been prescribed testosterone therapy. However, $2.7 \%$ had tried or currently used some medication other than testosterone in an effort to deal with unspecified erectile dysfunction.

Criticisms have been expressed concerning the ADAM-questionnaire $[17,18]$ as a screening tool. The questionnaire was tested in Chile in a group of 96 men age 40 or older, and was shown to have $83.3 \%$ sensitivity and $19.7 \%$ specificity in the detection of $\mathrm{LOH}$. Item 1 , "decreased sexual desire", was a better predictor of LOH than the results from the complete questionnaire (63.3\% sensitivity and $66.7 \%$ specificity) [28]. According to this $[25,26]$ and partly supported by our results concerning "less strong erections" and low BT levels in 45 YY men, is it reasonable to clinically suspect hypogonadism when symptoms of sexual dysfunction are present. We also tried to find a symptom complex suggesting hypogonadism by making extensive cluster analyses of aging men; both in the current study and in a previous study of 55-75 YY men [12], but without any success. However, 6 of 10 symptoms from the ADAM-questionnaire were more common in the $45 \mathrm{YY}$ men than in $35 \mathrm{YY}$ men, the causes of which could only be speculated on. Looking retrospectively, questions from the AMS-questionnaire may have given a better reliability in our questionnaire than the questions from the ADAM-questionnaire.

We were surprised by the low response-rate, which in earlier studies has been $73 \%$ to $87 \%$ [11-13]. It is possible that 35 and 45 year old men felt that the issues asked for were not relevant or suitable for them. It might also be that these age-groups of men are busier with such responsibilities as raising a family, being in the middle of their professional career, and having a very active leisure time. 
It was quite obvious that $35 \mathrm{YY}$ men and $45 \mathrm{YY}$ men were engaging in sports that were different from those in which older men take part in [11-13]. A total of $72 \%$ of $35 \mathrm{YY}$ and $65 \%$ of 45 YY men marked the ticked box "other sports", where it was possible to write in free text and name sports such as bodybuilding, floor hockey, downhill skiing, martial arts, soccer and/or ice-hockey were frequently mentioned. Perhaps we failed to detect some men engaging in sports due to this. However, from a "big picture" perspective, strength of our study is that we have used the same questionnaire in the current study as in our earlier studies of older men [11-13], since it is easy to compare the results between ages. We could, however had added some new alternatives to the list of different sports.

Comparing the results of the current study with the results from the study investigating 370 55-75 YY men [12] it is obvious that the prevalence of all but two of the symptoms from the ADAM-questionnaire increases in frequency with age. The two symptoms that were more common in the current study, "decreased enjoyment of life" and "sadness and/or grumpiness", are symptoms that are inversely connected to wellbeing. It might be that younger Swedish men are being stressed by their life-style, and experience this as a feeling of being insufficient in their everyday life with family, career, active leisure time etc. Besides decreasing androgen levels psychological stress could also reflect the high percentage of reported symptoms such as "deterioration in work performance" and/or "ability to play sports". Also the symptom burden was higher in older men [12], 85\% of 55-75 YY men were "ADAMpositive", more in the older age groups than in the youngest. In the current study, slightly more than half of 45 YY and about $40 \%$ of 35 YY men were "ADAM-positive”. It also seems that the symptom-burden increases according to the "ADAM-sum". More men report higher “ADAM-sum” with increasing age, from 35 YY to 60-65 YY. We have previously commented that 66-75 YY men report less symptom-burden than 60-65 YY men, perhaps having to do with fewer demands due to social causes associated with, for example, retirement [12] or perhaps that among elderly people, the healthiest men are the survivors and therefore 
more prone to answer the questionnaire than sedentary elderly men. Making comparisons between testosterone levels is very difficult due to the many different analysis methods, and unfortunately the methods of measuring T have changed slightly between 2007 and now, which is to say that the values from the current study may be somewhat different from those from the previous studies $[12,13]$. However, comparisons between the old method and the new one, RocheTestosterone II (2nd generation) have shown very similar results $(r=0.98)$ in regression analyses, making comparisons possible between the current and the previous studies $[12,13]$. It appears that T concentrations in $45 \mathrm{YY}$ men were lower, both than $35 \mathrm{YY}$ men in the current study and than the results from the older one [12]. This could be due to a selection bias, since more 45 YY men that gave blood-samples reported higher ADAMsum (although not significant) than those who did not give a blood-sample. Other reasons may also be involved, such as stress impairing testosterone production more in the 45 years old group than in a group of older retired men.

The 35 YY men who consume alcohol weekly or daily had higher BT than 45 YY. It can be speculated that ten years of weekly to daily intake of alcohol may lower BT concentrations. It should be pointed out, however, that the number of men included in this analyse was small, and therefore no wide conclusions can be drawn.

In summary; BT and T were lower in $45 \mathrm{YY}$ men than in $35 \mathrm{YY}$ men and, as expected, many testosterone deficiency symptoms were more common in the older age-group. It was surprising that $\mathrm{T}$ concentrations were lower in $45 \mathrm{YY}$ men than in 55-75 $\mathrm{YY}$ men from the former study [12]. However, this could be due to a selection bias, because 45 YY old men who gave blood samples had perhaps more symptoms. Hot flushes were more common among men who used psychoactive drugs, and this was not reported in our earlier studies using the same questionnaire [11-13]. The study was unfortunately limited by the low rate of response from the study population (38.7\%) and that we obtained blood-samples from slightly less than half of 200 chosen men, even though they had been asked to provide a sample and had agreed 
beforehand. This probably reflects the situation in which $35 \mathrm{YY}$ and $45 \mathrm{YY}$ men find themselves, in the middle of their careers and the child raising period. It is therefore difficult to draw any general conclusions from this study, even though the results do add to the knowledge of possible symptoms of testosterone deficiency in men. 


\section{Declaration of interest statement:}

The authors report no declarations of interest. 


\section{References}

1) Nahoul K, Roger M. Age-related decline of plasma bioavailable testosterone in adult men. J Steroid Biochem 1990;35:293-299.

2) Gray A, Feldman HA, McKinlay JB, Longcope C. Age, disease, and changing sex hormone levels in middle-aged men: results of the Massachusetts male aging study. J Clin Endocrinol Metab 1991;73:1016-1025.

3) Lunenfeld B, Nieschlag E. Testosterone therapy in the aging male. Aging Male 2007;10: $139-153$.

4) Morales A, Heaton JP, Carson CC III. Andropause: a misnomer for a true clinical entity. J Urol 2000;163:705-712.

5) Wang C, Nieschlag E, Swerdloff R, Behre HM, Hellstrom WJ, Gooren LJ, Kaufman JF, Legros JJ, Lunenfeld B, Morales A, Morley JE, Schulman C, Thompson IM, Weidner W, Wu FC. ISA, ISSAM, EUA, EAA, and ASA recommendations: investigation, treatment, and monitoring of late-onset hypogonadism in males. Aging Male 2009;12:5-12.

6) Morales A, Schulman C, Tostain J, Wu F. Testosterone Deficiency Syndrome (TDS) Needs to be Named Appropriately - The Importance of Accurate Terminology. Eur Urol 2006;50: 407-409.

7) Morley JE, Kaiser FE, Sih R, Hajjar R, Perry HM 3rd. Testosterone and frailty. Clin Geriatr Med 1997;13:685-695.

8) Morley JE, Perry HM. Androgen deficiency in ageing men. Med Clin North Am 1999;83:1279-1289.

9) Tan RS, Bransgrove L. Testosterone replacement therapy. What is its potential in elderly men? Postgrad Med 1998;103:247-248, 251-256.

10) Vermeulen A. Androgens in the aging male. J Clin Endocrinol Metab 1991;73:221-224. 
11) Spetz AC, Fredrikson MG, Hammar ML. Hot flushes in a male population aged 55, 65, and 75 years, living in the community of Linkoping, Sweden. Menopause 2003;10:81-87.

12) Spetz AC, Palmefors L, Skobe S, Strömstedt M, Fredriksson M, Theodorsson E, Mats Hammar. Testosterone correlated to symptoms of "Partial Androgen Deficiency in Aging Men” (PADAM) in a Swedish elderly population. Menopause 2007;14:999-1005.

13) Spetz Holm AC, Fredrikson MG, Theodorsson E, Palmefors LG, Karlsson PS, Joborn C, Hammar ML. Change in testosterone concentrations over time is a better predictor than the actual concentrations for symptoms of late onset hypogonadism. Aging Male. 2011 Jun 1. (Epub ahead of print).

14) Hanus M, Matouskova M, Starka L, Hill M. Hormonal homeostasis in a group of 216 Czech males and correlation with responses to a questionnaire of the University of St Louis. Aging Male 2006;9:103-110.

15) T’Sjoen G, Goemaere S, De Meyere M, Kaufman J. Perception of males`aging symptoms, health and well-being in elderly community-dwelling men is not related to circulating androgen levels. Psychoneuroendocrinology 2004;29:201-214.

16) Gladh M, Rahgozar M, Hammar M, Fredriksson M, Spetz A-C. Prevalence of symptoms possibly related to PADAM, in a Swedish population aged 55, 65 and 75 years. Maturitas 2005;50:161-166.

17) Morley JE. Sex hormones and diabetes. Diabetes Metab Rev 1998;6:6-15.

18) Morley JE, Charlton E, Patrick P et al. Validation of a screening questionnaire for androgen deficiency in aging males. Metabolism 2000;49:1239-1242.

19) Vermeulen A. The physical state of testosterone in plasma. In: V. H. T. James V. H. T. et al, editors. The Endocrine Function of the Human Testes. New York, NY: Academic Press; 1973. P. 157-170. 
20) Arver S, Lehtihet M. Current guidelines for the diagnosis of testosterone deficiency. Jones TH(ed): Advances in the management of testosterone deficiency. Front Horm Res. Basel, Karger 2009;37:5-20.

21) Corona G, Mannucci E, Petrone L et al. ANDROTEST: a structured interview for the screening of hypogonadism in patients with sexual dysfunction. J Sex Med 2006;3:706-715. 22) Heineman LAJ, Zimmerman T, Vermeulen A, Thiel C. A new "aging male" symptoms`(AMS) rating scale. Aging Male 1999;2:105-114.

23) Smith KW, Feldman HA, Kinlay JB. Construction and field validation of a selfadministered screener for testosterone deficiency (hypogonadism) in ageing men. Clin Endocrinol (Oxf) 2000;53:703-711.

24) Wu FCW, Abdelouahid T, Beynon J, Pye SR, Phil M, Silmam AJ, Finn JD, O`Neill TW, Bartfai G, Casanueva FF et al. Identification of late-onset hypogonadism in middle-aged and elderly men. N Engl J Med 2010;363:123-35.

25) Arujo AB, Esche GR, Kupelian V, O`Donnell AB, Travison TG, Williams RE, Clark RV, McKinlay JB. Prevalence of symptomatic Androgen deficiency in men. J Clin Endocrinol Metab 1992: 4241-4247; 2007.

26) Bhasin S, Cunningham GR, Hayes FJ, Matsumoto AM, Snyder PJ, Swerdloff RS, Montori VM. Testosterone therapy in adult men with androgen deficiency syndromes: an endocrine society clinical practice guideline. J Clin Endocrinol Metab 2006; 91:1995-2010.

27) Blumel JE, Chedraui P, Sebastian AG, Navarro A, Valenzuela K, Vallejo S. Is the androgen deficiency of aging men (ADAM) questionnaire useful for the screening of partial androgenic deficiency of aging men? Maturitas 2009;63:365-368.

28) Albertazzi P. Non-estrogenic approaches for the treatment of climacteric symptoms. Climacteric 2007;10 (suppl 2):115-120. 